\title{
Breve reseña sobre la Ley española 9/2014, General de Telecomunicaciones
}

\author{
DANIEL PÉREZ 1
}

\section{RESUMEN}

A nadie es ajeno el permanente avance y desarrollo al que se enfrenta el sector de las telecomunicaciones, que hace que el derecho deba estar en la capacidad de adaptarse a las necesidades y vicisitudes de esta materia. Ahora bien, España ha iniciado esta travesía por "regular lo difícilmente regulable" con la dificultad adicional de compaginar sus políticas, planes, programas y proyectos con el régimen de la Unión Europea, obteniendo como resultado de ello la Ley 9. de 9 de mayo de 2014, General de Telecomunicaciones.

Palabras clave: telecomunicaciones, España, Ministerio de Industria, Energía y Turismo, Comisión Nacional de los Mercados y la Competencia.

\section{Comments on the spanish telecommunications Act 9 of 2014}

\section{ABSTRACT}

The continuous progress and development experienced by the telecommunications sector is not strange to anyone. This is why the law should adapt to the needs and issues pertaining to the sector. Spain has undertaken efforts in

1 Monitor del Departamento de Derecho de las Telecomunicaciones, Universidad Externado de Colombia, Bogotá, Colombia. Judicante de la Sección Primera del Consejo de Estado (2015). Correo electrónico: daniel.castaneda@est.uexternado.edu.co Fecha de recepción: 19 de agosto de 2015. Fecha de modificación: 20 de septiembre de 2015. Fecha de aceptación: 10 de octubre de 2015. Para citar el artículo: PÉREZ, D. (2015). Breve reseña sobre la Ley española 9/2014, General de Telecomunicaciones. Revista Digital de Derecho Administrativo n. ${ }^{\circ}$ 14, Universidad Externado de Colombia, pp. 259-277. DOI: http://dx.doi. org/10.18601/21452946.n14.13 
order to "regulate difficult regulatory matters", and to reconciling its policies, plans, programs and projects with those within the European Union regime. These attempts have turned out to be the General Law of Telecommunications of 9 May 2014.

Keywords: Telecommunications, Spain, Ministry of Industry, Energy and Tourism, Committee of the National Markets and Competition.

\section{ANTECEDENTES}

Para el año 2009, el sector de las telecomunicaciones en la Unión Europea (UE) sufría diferentes problemáticas tales como la fragmentación regulatoria y la incoherencia entre las competencias y actividades desarrolladas por las distintas Autoridades Nacionales de cada uno de los países miembros, lo que suponía un peligro inminente para el sector mismo y sus usuarios, a la vez que impedía alcanzar su principal objetivo, la consolidación de un mercado único de comunicaciones electrónicas.

Es por ello que el Parlamento y el Consejo Europeo expidieron la Directiva 2009/140/CE² de 25 de noviembre de 2009, con la cual se pretendió modificar el Marco Regulador de la Unión Europea de las redes y los servicios de comunicaciones electrónicas expedido en el año $2002^{[3]}$, para así compaginarlo con la constante y dinámica invocación de este importante sector de la economía, todo ello en pos de lograr una regulación coordinada y armonizada de orden comunitario.

Los aspectos relevantes de esta transcendental reforma fueron: (i) La búsqueda de una sociedad de la información asequible y de alta calidad para todos los usuarios finales; (ii) Una gestión coordinada y eficiente del espectro; (iii) La prevalencia de las leyes del mercado y la competencia, tanto internas como internacionales; (iv) La introducción de la neutralidad tecnológica tendiente al desarrollo de una competencia leal entre los sujetos que intervienen en el

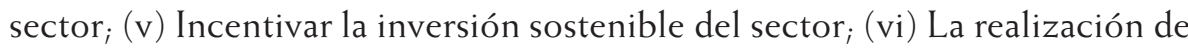
una adecuada actualización conceptual de acuerdo a las necesidades mismas que arroja el sector; (vii) El refuerzo de la independencia de las autoridades

2 "Por la que se modifican la Directiva 2002/21/CE relativa a un marco regulador común de las redes y los servicios de comunicaciones electrónicas, la Directiva 2002/19/CE relativa al acceso a las redes de comunicaciones electrónicas y recursos asociados, y a su interconexión, y la Directiva 2002/20/CE relativa a la autorización de redes y servicios de comunicaciones electrónicas".

3 Compuesto por: (i) Directiva 2002/21/CE, también conocida como la Directiva Marco (ii) Directiva 2002/19/CE o Directiva sobre acceso; (iii) Directiva 2002/20/CE o Directiva sobre autorización, (iv) Directiva 2002/22/CE o Directiva sobre servicio universal, y (v) Directiva 2002/58/CE o Directiva sobre la privacidad y las comunicaciones electrónicas. 
nacionales con el ánimo de garantizar una aplicación efectiva de este nuevo marco regulador.

Posteriormente, en el año 2010, la Comisión Europea elaboró un documento $^{4}$ en el que se expone el alcance de la Agenda Digital para Europa, documento que se compartió con las demás autoridades de la Unión, a saber, el Parlamento Europeo, el Consejo de Europa, el Comité Económico y Social Europeo y el Comité de las Regiones.

La Agenda Digital para Europa es una visionaria iniciativa, dentro de la estrategia desarrollada por la Comisión Europea, que se concreta en el texto "Europa $2020^{\prime \prime}$, con la que se pretende hacer volver por la buena senda a la economía europea, tan golpada al finalizar la primera década del siglo XXI, y prepararla para la nueva década, mediante el aprovechamiento efectivo del potencial económico y social de las TICs, especialmente de internet ${ }^{6}$. Para ello se propusieron acciones como las siguientes:

- La apertura al acceso de contenidos a través de:

a. La simplificación de autorización, gestión y concesión de licencias sobre derechos de autor, y

b. La simplificación en las transacciones en línea y transfronterizas.

- Reforzar el Marco Regulador de Protección de Datos de la UE ${ }^{7}$, por medio de la adopción de marcas de confianza en línea, mecanismos de solución

4 Comunicación de la Comisión al Parlamento Europeo, al Consejo, al Comité Económico y Social Europeo y al Comité de las Regiones. "Una Agenda Digital para Europa". Comisión Europea. Bruselas, 26 de agosto de 2010.

5 "Europa 2020 expone una estrategia para conseguir unos niveles elevados de empleo, una economía de baja emisión de carbono, productividad y cohesión social, que debe aplicarse a través de medidas concretas a nivel nacional y de la UE. Esta batalla por el crecimiento y el empleo exige una toma de conciencia en las altas esferas políticas y la movilización en toda Europa de la totalidad de los agentes". Ibíd., p. 3.

6 El documento reconoce como falencias de la economía respecto del sector (i) la fragmentación de los mercados digitales; (ii) la fragmentación y disonancia regulatoria; (iii) la ausencia de inversión y, por ende, (iv) la ausencia de investigación e innovación; y (v) la falta de capacitación y educación digital, es decir, la falta de conocimiento sobre el manejo y beneficios que reportan las TICs al desarrollo de la vida cotidiana, lo que genera de manera concatenada (vi) desconfianza digital.

7 Se destaca el acuerdo de los Ministros de las Unión Europea sobre el futuro Reglamento Europeo en materia de Protección de Datos. "El objeto de este documento es lograr un equilibrio entre la privacidad de las empresas, beneficiando a las pequeñas y medianas empresas, el desarrollo tecnológico y los ciudadanos. La aprobación de dicho reglamento, implicará que la Unión Europea cuente con un marco más riguroso y coherente en materia de protección de datos. Asimismo, se refuerza el derecho al olvido y los tres derechos que se derivan, como lo son el de ser borrado, el de olvido y el de portabilidad de los datos". Tomado de Boletín e-Telecomunicaciones. Edición 20. Bogotá, Colombia. Universidad 
de controversias en línea y el establecimiento de un código de derechos de los usuarios.

- Revisar la política europea de normalización, no solo en telecomunicaciones sino también en materias conexas como propiedad intelectual.

- Garantizar cobertura universal de banda ancha con velocidades crecientes, y fomentar el despliegue de infraestructura necesaria para ello.

- Fomentar y coordinar la inversión pública y privada.

- Impulsar de educación digital.

Ahora bien, con fundamento en lo anterior y con el fin de dar cumplimiento a la obligación de incorporar y adecuar el marco normativo de España, el Gobierno español presentó en 2011 un primer Proyecto de Ley por la que se modifica la Ley 32/2003, de 3 de noviembre, General de Telecomunicaciones. Sin embargo, este proyecto no tuvo un afortunado desenlace.

No obstante ello, producto de la expedición de la Ley 3 a $^{a}$ de 4 de junio de 2013, el marco institucional en materia de telecomunicaciones sufrió modificaciones, toda vez que por esta ley se creó la Comisión Nacional de los Mercados y la Competencia ${ }^{8}$, un organismo de carácter público con personalidad jurídica y plena capacidad pública y privada para actuar, al que se le encomendó la labor de "garantizar, preservar y promover el correcto funcionamiento, la transparencia y la existencia de una competencia efectiva en todos los mercados y sectores productivos, en beneficio de los consumidores y usuarios", labor que entraría a desempeñar incluso dentro de mercado de las comunicaciones electrónicas ${ }^{10}$, lo que hizo necesario replantear la distribución de competencias entre autoridades del sector, atendiendo asimismo que la nueva ley suprimió la Comisión del Mercado de Telecomunicaciones ${ }^{11}$.

Este nuevo marco competencial previsto en la ley ${ }^{12}$ incidió en el proceso de formación de un nuevo proyecto de ley en materia de comunicaciones electrónicas, el cual no solo incorporaría esta trascendental modificación, sino también los cambios agregados en las Directivas de 2009 y las modificaciones introducidas por el Real Decreto-Ley 13 de 30 de marzo de 2012, por el que

Externado de Colombia. [Fecha de consulta: 30 de agosto de 2015]. Disponible en: http:// documentos.uexternado.edu.co/78435129/wp-content/uploads/2015/08/BOLETIN-20.pdf

8 Sobre el particular cfr.: Carlón Ruiz (2014), Montero Pascual (2013) y Ortiz Laverde (2014).

9 Ley 3 a $^{\text {a de }} 04$ de junio de 2013, de creación de la Comisión Nacional de los Mercados y la Competencia. Artículos 1 y 2.

10 Ley $3 .{ }^{a}$ de 4 de junio de 2013 . Artículo 6.

11 Ver Anexos: Cuadro de traslado de competencias de la Comisión del Mercado de las Telecomunicaciones (hoy Comisión Nacional de los Mercados y la Competencia) al Ministerio de Industria, Energía y Turismo.

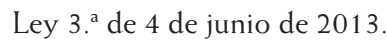


se transponen directivas en materia de mercados interiores de electricidad y gas y en materia de comunicaciones electrónicas, y por el que se adoptan medidas para la corrección de las desviaciones por desajustes entre los costes e ingresos de los sectores eléctrico y gasista.

Finalmente en el año 2013, al inicio de la legislatura, el Consejo de Ministros aprobó la remisión a las Cortes Generales de un nuevo Proyecto de Ley General de Telecomunicaciones después de que se hubieran surtido los trámites por parte de las distintas autoridades, como lo eran los informes de la Comisión del Mercado de Telecomunicaciones ${ }^{13}$ y los informes sobre el Anteproyecto de Ley de creación de la Comisión Nacional de los Mercados y la Competencia (MTZ 2012/398), del Consejo Asesor de las Telecomunicaciones y de la Sociedad de la Información (Informe de 21 de enero de 2013) y del Consejo de Estado (Dictamen 468 de 24 de julio de 2013) ${ }^{14}$.

\section{COMPOSICIÓN NORMATIVA}

La Ley española 9 de 9 de mayo de 2014, General de Telecomunicaciones, sancionada por el Rey Juan Carlos I, está compuesta por ocho títulos que agrupan un total de ochenta y cuatro artículos ${ }_{i}$ junto a ellos se establecen: diecinueve disposiciones adicionales, doce disposiciones transitorias, una disposición

13 El informe elaborado por la Comisión del Mercado de Telecomunicaciones indicó que la distribución competencial que se realizaba en el anteproyecto era contraria a la práctica comunitaria, en el entendido de que las funciones de naturaleza administrativa, como llenar los registros de los operadores y asignar la numeración, tienen una incidencia directa con la labor del regulador, y por consiguiente dichas funciones deberían permanecer en cabeza de un solo ente, en este caso la Comisión Nacional de los Mercados y Competencia, y no de una autoridad administrativa como el Ministerio de Fomento o la Secretaría de Telecomunicaciones.

14 En el mencionado informe se ratifica lo expuesto por parte de la Comisión del Mercado de Telecomunicaciones, en lo referente a la distribución competencial. Sobre el particular se debe tener en cuenta la Comunicación remitida por la Comisión Europea sobre la incidencia que podría tener el marco legal desarrollado en el proyecto de creación de la Comisión Nacional de los Mercados y la Competencia y en el anteproyecto de Ley General de Telecomunicaciones, donde se indica que la integración no solo afectaría la independencia del regulador, sino la labor que este realizaba. ORTIZ LAVERDE (2014, pp. 618, nota 1593) sobre el particular indica: "Quienes fueron muy críticos en la distribución realizada, a pesar de precisar que esta se ha adecuado a los criterios previstos en el seno de la Unión Europea y que es de plena libertad del legislador realizar un reparto competencial sobre las funciones que inicialmente estaban asignadas a un órgano para ser asumidas por otro órgano, haciendo hincapié que las competencias asignadas a la CNMC corresponde a una visión restringida de regulación y lo que implica la regulación ex ante implica la confluencia de varios elementos y funciones de carácter administrativo indispensables para el monitoreo permanente del mercado". 
derogatoria, once disposiciones finales y dos anexos; todo ello precedido por un prólogo en el que se hace una rápida exposición de la ley.

Estructura que se presenta como atípica si se compara con la legislación colombiana, pero que, en nuestro criterio, muestra un mayor grado de organización y sistematización normativa.

\section{COMPOSICIÓN TEMÁTICA}

\section{EXPosición DE Motivos DE LA LeY}

La Ley 9/2014, en su exposición de motivos, trae una breve presentación de los pilares estructurales sobre los cuales se erigió la regulación contenida en ella.

Toma como punto de partida la trascendencia de las telecomunicaciones como uno de los sectores más dinámicos de la economía actual, por el hecho de contar con la potencialidad de invertir de manera directa en el desarrollo económico y el bienestar social, tanto de España como de la comunidad en general, siendo conocida la capacidad del sector de las telecomunicaciones de repercutir de manera positiva en el crecimiento económico a partir del mejoramiento de la productividad y la disminución del desempleo. Se plantean como pilares de la ley:

1. La inversión, ya que es innegable que el sector de las telecomunicaciones es un mundo de constante innovación que implica enormes y constantes aportes de capital.

2. La competencia efectiva, pues serán los parámetros de la libre y efectiva competencia aplicados sobre las fuerzas del mercado los mecanismos idóneos que incidirán en el precio, la calidad e innovación de los bienes y servicios.

3. La recuperación de la unidad de mercado en el sector de las telecomunicaciones. Este pilar, reconocido por la misma ley como una necesidad, se cumplirá, por un lado, mediante el establecimiento de procedimientos de coordinación y resolución de conflictos entre la regulación de orden estatal y la expedida por las distintas administraciones competentes, lo que traerá repercusiones en materia de despliegue de redes y prestación de servicios; y por el otro, por medio de una simplificación administrativa, consistente, por ejemplo, en (i) la eliminación de licencias y autorizaciones para determinadas categorías de instalaciones para el uso del espectro, facilitando el despliegue de nuevas redes, y (ii) la reducción de cargas administrativas a los operadores, como la consistente en la eliminación de ciertas obligaciones en cabeza de estos últimos.

4. El refuerzo sobre controles y potestades de inspección y sanción, facilitando así la adopción de medidas cautelares y revisando las cuantías de las sanciones. 
Ahora bien, teniendo claras las bases de la ley, se hace más sencillo captar la esencia de las pretensiones de la misma, entre las cuales tenemos: (i) determinar condiciones necesarias para (a) la existencia de competencia efectiva, (b) la realización de inversiones para el despliegue de redes de nueva generación y (c) la prestación de nuevos servicios, y (ii) brindar seguridad jurídica a operadores y usuarios.

\section{LOS TíTULOS}

\section{Título I. Disposiciones generales}

Lo primero que debe resaltarse de la Ley 9/2014 es el hecho de ampliar el espectro temático, para así no solo regular lo concerniente a las comunicaciones electrónicas sino regular las telecomunicaciones en general ${ }_{i}$ para ello, la misma ley se ha encargado de definir las telecomunicaciones como servicios de interés general que se prestan en el régimen de la libre competencia, tal como se indica en el Libro Verde sobre Servicios de Interés General ${ }^{15}$.

En este punto, la ley reafirma algunos de los pilares bajo los cuales se estructura, pues dirige tanto los objetivos como los principios ${ }^{16}$ de la misma hacia el fomento de la competencia efectiva, la salvaguarda del mercado de las telecomunicaciones y la promoción de la inversión, claro está, sin ser los únicos, pues se destacan también el desarrollo del sector y de la economía en general, la protección a los usuarios y la facilitación del acceso a los servicios, entre otros.

Igualmente, la ley es clara en determinar qué materias del sector no cobija pero son objeto de regulación por otras leyes, motivo por el cual excluye expresamente:

- La regulación de contenidos difundidos a través de servicios de comunicación audiovisual $^{17}$, si bien aclara que las redes utilizadas como soporte de radiodifusión sonora y televisiva y recursos asociados sí son de su competencia.

- La prestación de servicios sobre las redes de telecomunicaciones que no consistan principalmente en el trasporte de señales por dichas redes ${ }^{18}$.

15 Green Paper in Services of General Interest. Commission of the European Communities. Bruselas, 21.5.2003. COM(2003) 270 final. Disponible en: http://europa.eu/legislation_summaries/competition/state_aid/123013_en.htm

16 Entre ellos podemos citar los principios de igualdad, no discriminación, eficiencia, eficacia, neutralidad tecnológica y universalización, entre otros.

17 Temática dejada en manos del Régimen de Medios de Comunicación Social.

18 Temática dejada en manos de la Regulación sobre la Sociedad de la Información y Comercio Electrónico. 


\section{Título II. Régimen general de explotación de redes y prestación de servicios de telecomunicaciones}

En este título, lo primero que cabe resaltar es que la explotación de redes y prestación de servicios de telecomunicaciones se hace bajo los precios de libre competencia, la legalidad, el respeto de derechos y las libertades fundamentales $^{19}$, entre otros.

En segundo lugar, la ley concede una habilitación de carácter general para la explotación redes, con el único requisito de notificar de ello al Registro de Operadores; registro de naturaleza pública manejado por el Ministerio de Industria, Energía y Turismo ${ }^{20}$, y cuyo procedimiento quedará sujeto a la regulación que se expida a propósito vía real decreto.

En este título se consagran las condiciones de existencia que deben y deberán cumplir los operadores públicos (operadores controlados por administraciones públicas), quienes deberán actuar bajo el principio de inversor privado.

Se establece la obligación de suministro de información ${ }^{21}$ a aquellas personas, físicas o jurídicas, que exploten redes o presten servicios de telecomunicaciones; información que será solicitada por las Autoridades Nacionales de Regulación (en adelante ANR) de las telecomunicaciones, para diversas finalidades que van desde necesidades estadísticas, pasando por la verificación del cumplimiento de condiciones y obligaciones, hasta la planeación en materia presupuestaria.

También se fomenta la aplicación de las normas técnicas que se dicten en la Unión Europea, en dirección a la idea de armonización de servicios; ahora bien, en el caso de no haber norma técnica sobre un tema se dará aplicación a las normas y/o recomendaciones que expidan las diferentes organizaciones

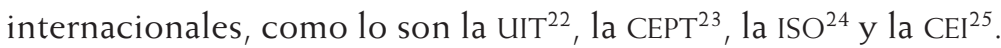

19 No solo las consagradas en la Constitución Española, sino también en los principios generales del derecho comunitario, en la Carta de Derechos Fundamentales de la Unión Europea y en el Convenio Europeo para la Protección de los Derechos Humanos y de las Libertades Fundamentales.

20 Esta habilitación fue introducida en paquete de telecomunicaciones expedido en el año 2002, en el que se incorporó la habilitación general para la prestación de los servicios de telecomunicaciones. Aspecto que fue consagrado en la Ley 32 de 2003, Ley General de Telecomunicaciones, vigente hasta el año 2014

21 Obligación que se incorpora expresamente en la Ley 32 de 2003. Lo anterior atendiendo a la importancia que tiene la información de los operadores para el ejercicio de las funciones encomendadas al regulador, particularmente en lo que se refiere a la definición de los mercados, los mercados susceptibles de regulación ex ante y la definición de operadores con posiciones de dominio.

Unión Internacional de Telecomunicaciones.

23 Conferencia Europea de Administración de Correos y Telecomunicaciones.

24 Comisión Internacional de Normalización.

25 Comisión Electrotécnica Internacional. 
En materia de interconexión, se pone de presente que los operadores de redes públicas tendrán el derecho y la obligación (i) de negociar sin restricción alguna y (ii) de la interconexión mutua para la prestación del servicio de telecomunicaciones; temáticas que quedarán sometidas al desarrollo reglamentario que se expida a propósito.

En materia de numeración, direccionamiento y denominación, corresponde al Ministerio de Industria, Energía y Turismo el otorgamiento y la cancelación de derechos de uso de recursos públicos de numeración, direccionamiento y denominación de acuerdo a los planes nacionales que para ello se expidan, competencia que bajo el régimen previsto en la Ley 32/2003 estaba en cabeza de la extinta Comisión del Mercado de Telecomunicaciones.

\section{Título III. Obligaciones y derechos de operadores y usuarios}

En lo relativo a las obligaciones de los operadores, estos estarán sujetos al régimen de las obligaciones de servicios públicos y de carácter público; es de anotar que la misma ley hace una categorización de estas obligaciones y habla entonces de:

- Una primera categoría, denominada obligaciones de servicio universal, entendida como la garantía de la prestación del servicio a todos los usuarios finales con independencia de su ubicación geográfica, con una calidad determinada y a precios asequibles ${ }^{26}, \mathrm{y}$

- Una segunda, las otras obligaciones, que se entenderá compuesta por obligaciones que imponga el Gobierno por distintas razones como seguridad, cohesión territorial, facilitación del servicio, entre otras.

El control sobre ambas categorías queda en manos del Ministerio de Industria, Energía y Turismo, nuevas competencias asumidas por esta autoridad administrativa, bajo la nueva distribución competencial realizada por la Ley 3/2013 y la Ley $9 / 2014$.

De los derechos de los operadores podemos resaltar (i) el derecho de ocupación del dominio público como del privado ${ }^{27}$, (ii) el derecho al uso compartido de la propiedad pública o privada, (iii) el derecho al despliegue de redes y (iv) el derecho al acceso a infraestructura de otros sectores.

Corresponde a los operadores garantizar el secreto de las comunicaciones; cualquier clase de interceptación deberá ser acorde a los parámetros que para ello consagran la Ley de Enjuiciamiento Criminal, Ley Orgánica 2/2002, sobre

26 Esta garantía es un desarrollo de lo previsto en la Directiva 2002/22/CE de Servicio Universal.

27 Previstos para casos en los que medie necesidad para la instalación de redes. 
el control judicial previo del Centro Nacional de Inteligencia, entre otras normas también de carácter orgánico. Igualmente, la Ley 9/2014 dispone que corresponde a los operadores adoptar medidas de protección de datos de carácter personal, las cuales podrán ser evaluadas por la Agencia Española de Protección de Datos.

Respecto a los derechos de los usuarios finales, tenemos (i) el derecho a celebrar contratos con los operadores, (ii) el derecho a resolver los mismos, (iii) el derecho a cambio de operador, (iv) el derecho a la continuidad del servicio y a la compensación inmediata por interrupción del mismo, (iv) el derecho a la información, (v) el derecho de acceso a servicio de emergencia y (vi) el derecho a elegir medio de pago, entre otros; todos ellos aunados a los previamente reconocidos en la Ley General para la Defensa de los Consumidores y Usuarios ${ }^{28}$.

Finalmente, dentro de este título la Ley 9/2014 dispone la existencia de mecanismos extrajudiciales de resolución de controversias entre operadores y usuarios finales, cuando estos son personas físicas y las controversias se refieren específicamente a sus derechos como usuarios; al respecto, valga aclarar que si bien es cierto la ley en estudio no especificó qué autoridad conocerá de tales controversias, sí dijo que será el Ministerio de Industria, Energía y Turismo quien determinará el procedimiento aplicable.

\section{Título IV. Normalización técnica y condiciones de los instaladores}

Dispone la Ley 9/2014 que todo establecimiento de condiciones relativas a la publicación y elaboración de las especificaciones técnicas tanto de interfaces ofrecidas en España como de equipos y aparatos de telecomunicaciones se hará vía real decreto; dichas condiciones serán establecidas con el ánimo de garantizar el cumplimiento de los requisitos esenciales en los procedimientos de evaluación.

Igualmente, en dicho decreto podrán ser determinados aquellos dispositivos que no se encuentran sujetos a las medidas, como también el procedimiento para la retirada del mercado de los productos que incumplan la normativa.

Toda esta reglamentación podrá ser complementada por el Ministerio de Industria, Energía y Turismo.

También estará sujeto a regulación vía decreto lo relacionado con los requisitos para el desarrollo de actividad de instalación o mantenimiento de equipos y sistemas de telecomunicaciones, donde el interesado en prestar dicho servicio, previo inicio de la actividad, deberá presentar declaración responsable sobre el cumplimiento de requisitos para prestar la actividad aquí referida al Registro de Empresas Instaladoras de Telecomunicación. 


\section{Título V. El dominio público radioeléctrico}

El espectro electromagnético es un bien de dominio público cuya titularidad está en cabeza del Estado. Su administración se hará teniendo de presente su importancia social, cultural y económica, y estará sujeta no solo a la normativa a propósito que contemple el ordenamiento español, sino también la normativa de la Unión Europea y las resoluciones y recomendaciones de la UIT y demás autoridades en la materia, ello con el ánimo de armonizar el uso del espectro radioeléctrico dentro de la Unión.

La Ley 9/2014 clasifica el uso del espectro en uso común, uso especial y uso privativo.

Respecto de los títulos habilitantes para explotar este valioso recurso, la ley en estudio consagra tres tipos de autorizaciones: a) autorizaciones generales, b) autorizaciones individuales y c) afectaciones administrativas ${ }^{29-30}$; títulos que se extinguen por causales contenidas en la misma ley, entre ellas, sin ser las únicas, la muerte del titular del derecho, la renuncia del titular al derecho, la pérdida de la condición de operador y la falta de pago de las tasas por reserva del dominio público.

Igualmente, queda habilitado el Ministerio de Industria, Energía y Turismo para revocar dichos títulos habilitantes, mediante el procedimiento establecido en el Régimen Jurídico de las Administraciones Públicas y del Procedimiento Administrativo Común ${ }^{31}$.

Finalmente, se dio facultad a la Secretaría de Estado de Telecomunicaciones y para la Sociedad de la Información, para que en cualquier tiempo pueda realizar la protección activa del espectro mediante emisiones no sustantivas en aquellas frecuencias que no han sido otorgadas a los operadores.

29 También denominadas dentro de la misma ley como concesiones administrativas.

30 La autorización general se concederá en los supuestos de uso especial (explotación de forma compartida, sin limitación de número de operadores o usuarios) de las bandas de frecuencia habilitadas a tal efecto a través de redes públicas de comunicaciones electrónicas instaladas o explotadas por operadores de comunicaciones electrónicas. Por su parte, el título habilitante revestirá la forma de autorización individual cuando: (i) Se trate de una reserva de derecho de uso especial por radioaficionados u otros sin contenido económico en cuya regulación específica así se establezca, o (ii) Se otorgue el derecho de uso privativo (explotación en exclusiva o por un número limitado de usuarios de determinadas frecuencias en un mismo ámbito físico de aplicación) para auto-prestación por el solicitante, salvo en el caso de administraciones públicas, que requerirán de afectación demanial, es decir, de afectación a bienes de dominio público. Finalmente, la afectación administrativa se usará en el resto de supuestos que no encajen en ninguna de las dos modalidades anteriores, siendo requisito necesario para el solicitante no estar incurso en ninguna de las prohibiciones para contratar contenidas en la Ley de Contratos del Sector Público (Real Decreto Legislativo 3. ${ }^{\circ}$ de 14 de noviembre de 2011).

31 Ley 30 de 26 de noviembre de 1992. 


\section{Título VI. Administración de las telecomunicaciones}

Este título se encuentra compuesto por tres artículos en los que se fijan las competencias, en materia de telecomunicaciones, de la Administración General del Estado, del Ministerio de Industria, Energía y Turismo y de la Comisión Nacional de los Mercados y la Competencia.

Dispone el artículo 68 de la Ley 9/2014 que el Gobierno, el Ministerio de Industria, Energía y Turismo, el Ministerio de Economía y Competitividad, y la Comisión Nacional de los Mercados y la Competencia, tendrán la consideración de Autoridades Nacionales de Telecomunicaciones ${ }^{32}$, a las cuales corresponderá fomentar la aplicación coherente de la normativa comunitaria y apoyar activamente los objetivos y actividades del ORECE ${ }^{33}$.

Para ello, estas autoridades deberán:

- Promover un entorno regulador previsible y coherente.

- Fomentar la cooperación entre inversores y la inversión eficiente en el mercado y la innovación en infraestructuras.

- Imponer obligaciones específicas con el ánimo de mantener una competencia efectiva y sostenible.

- Garantizar la no discriminación entre empresas suministradoras de redes y servicios de telecomunicaciones.

- Salvaguardar la competencia en beneficio de los consumidores (usuarios).

A renglón seguido, la ley hace una distribución de funciones administrativas y regulatorias al tenor de sus artículos 69 y $70^{[34]}$, precisando claramente cuáles serán las competencias del Ministerio de Industria, Energía y Turismo, y cuáles de la Comisión de Nacional de Mercados y la Competencia. En este punto es sumamente necesario resaltar que el Ministerio en comento gozará de una competencia residual, puesto que quedará habilitado para asumir las competencias en materias de telecomunicaciones que no hayan sido atribuidas de manera expresa a la Comisión o a cualquier otro organismo ${ }^{35}$.

32 Respecto de los ministerios, se debe hacer la precisión de que la ley se refiere puntualmente a sus órganos superiores y directivos.

33 Organismo de Reguladores Europeos de las Comunicaciones Electrónicas.

34 Ver Anexos: Cuadro de funciones del Ministerio de Industria, Energía y Turismo y la Comisión Nacional de Mercados y la Competencia (arts. 69 y 70, de la Ley 9. ${ }^{a}$ de 09 mayo de 2014)

35 Ver Anexos: Cuadro de traslado de competencias de la Comisión del Mercado de las Telecomunicaciones (hoy Comisión Nacional de los Mercados y la Competencia) al Ministerio de Industria, Energía y Turismo. 


\section{Título VII. Tasas en materia de telecomunicaciones}

Esta ley también contiene regulación sobre las tasas en materia de telecomunicaciones; $;$ se dispone que estas son producto del poder impositivo del Estado bajo una óptica objetiva, transparente y proporcional, y que su finalidad será cubrir los gastos administrativos que se generen con ocasión de la gestión, control y ejecución del régimen establecido en la ley misma, al igual que los gastos de cooperación internacional, armonización, normalización y análisis de mercado.

\section{Título VIII. De la inspección y el régimen sancionador}

En materia de inspección, la ley ha determinado que dicha facultad radica en cabeza del Ministerio de Industria, Energía y Turismo y de la Comisión Nacional de Mercados y la Competencia, en donde a cada uno le correspondieron temáticas precisas; al Ministerio le corresponde la inspección de lo relacionado con (i) las condiciones de explotación y prestación de redes y servicios, (ii) los equipos y aparatos de las instalaciones y servicios civiles, (iii) el dominio público radioeléctrico y (iv) los servicios de tarifación adicional. Por su parte, corresponde a la Comisión la inspección sobre los operadores conforme a la competencia sancionadora dada a esta en su ley de creación ${ }^{36}$.

En lo que respecta a la competencia sancionadora contenida en la ley aquí reseñada, le corresponderá imponer las sanciones a (i) el Secretario de Estado de Telecomunicaciones y para la Sociedad de la Información ${ }^{37}$, (ii) la Comisión Nacional de Mercados y la Competencia y (iii) la Agencia Española de Protección de Datos.

Dentro de la tipología de sanciones contenidas en la ley se pueden encontrar multas, que oscilan entre los 50.000 euros y los 20 millones de euros, e inhabilidades, sea para instaladores u operadores.

\section{LAS DISPOSICIONES ADICIONALES}

A manera de disposiciones adicionales, la ley hace una serie de precisiones regulatorias sobre determinados temas, como por ejemplo, limitaciones a la propiedad y servidumbres, mecanismos de notificación y publicidad de actos, universalización de la banda ancha ultrarrápida y medidas de impulso de la innovación en el sector.

Igualmente, es en estas disposiciones que se hace mención:

36 Sobre las funciones inspectoras y sancionatorias de la Comisión Nacional de Mercados y la Competencia véase el artículo 29 de la Ley 3. ${ }^{a}$ de 4 de junio de 2013 en concordancia con el artículo 72 de la Ley 9. ${ }^{a}$ de 9 de mayo de 2014. 
i. Del Consejo Asesor de las Telecomunicaciones y de la Sociedad de la Información, un órgano asesor del Gobierno presidido por el Ministro de Industria, Energía y Turismo, y cuya estructura y funcionamiento quedan supeditados a la expedición de un real decreto a propósito.

ii. De la Comisión Interministerial sobre radiofrecuencias y salud, integrada por los Ministerios de Industria, Energía y Turismo; Sanidad, Servicios Sociales e Igualdad, y el Instituto de salud Carlos III por parte del Ministerio de Economía y Competitividad, cuya estructura y funcionamiento también se encuentran supeditados a la expedición de real decreto.

iii. De la Entidad Pública Empresarial Red.es, que tiene funciones relacionadas con la regulación sobre los dominios en internet.

\section{LAS DISPOSICIONES TRANSITORIAS}

Aquí se encuentran contenidas, como su nombre lo indica, un conjunto de disposiciones con las que se pretende empezar a dar aplicación a la Ley 9/2014, haciendo el tránsito de una regulación a otra y compaginando esta con la normativa vigente al momento de su inclusión en el ordenamiento jurídico español.

A manera de ejemplo, se regula aquí la continuidad de la normativa previa, el tratamiento de procedimientos previamente iniciados y un régimen transitorio de imposición de tasas.

\section{LA DISPOSICIÓN DEROGATORIA}

A partir de la entrada en vigencia de la Ley 9/2014 quedan derogadas: la Ley 11 de 24 de abril de 1998, General de Telecomunicaciones; la Ley 32 de 3 de noviembre de 2003, General de Telecomunicaciones, y toda disposición contraria, sea esta de igual o inferior jerarquía.

\section{LAS DISPOSICIONES FINALES}

En ellas se modifican o adicionan artículos de las siguientes leyes:

Decreto Ley 01 de 27 de febrero de 1998

Sobre infraestructura común en los edificios para el acceso de servicios de telecomunicación.

Ley 38 de 5 de noviembre de 1999

De Ordenación de la Edificación.

Ley 34 de 11 de julio de 2002

De servicios de la sociedad de la información y de comercio electrónico.

Ley 59 de 19 de diciembre de 2003

De firma electrónica. 
Ley 25 de 18 de octubre de 2007

De conservación de datos relativos a las comunicaciones electrónicas y a las redes públicas de comunicaciones.

Ley 7. ${ }^{a}$ de 31 de marzo de 2010

General de la Comunicación Audiovisual.

Ley 13 de 27 de mayo de 2011

De regulación del juego.

\section{LOS ANEXOS}

Por último, la ley cuenta con un par de anexos; en el primero de ellos se habla de las tasas en materia de telecomunicaciones, y puntualmente, de tasa general de los operadores, tasa por numeración, direccionamiento y denominación, tasa por reserva del dominio público radioeléctrico y tasas de telecomunicaciones, el segundo es un compendio de términos y definiciones utilizadas dentro de la ley, con el sentido que a cada uno le asigna la misma.

\section{REFERENCIAS}

Boletín e-Telecomunicaciones. Edición 20. Bogotá, Colombia. Universidad Externado de Colombia. [Fecha de consulta: 30 de agosto de 2015]. Disponible en: http://documentos.uexternado.edu.co/78435129/wp-content/uploads/2015/08/ BOLETIN-20.pdf

Boletín Oficial de las Cortes Generales. IX Legislatura. Serie A: Proyectos de Ley. 27 de mayo de 2011, n. ${ }^{\circ} 124-1$. Proyecto de Ley 121/000124 por la que se modifica la Ley 32/2003, de 3 de noviembre, General de Telecomunicaciones.

CARLÓN RUIZ, MATILDE. (2014). Reordenación de las competencias regulatorias en relación con el mercado de las comunicaciones electrónicas a resultas de la aprobación de las leyes $3 / 2013$, de creación de la CNMC, y 9/2014, General de Telecomunicaciones. La Comisión Nacional de los Mercados y la Competencia, pp. 299-364.

Comunicación de la Comisión al Parlamento Europeo, al Consejo, al Comité Económico y Social Europeo y al Comité de las Regiones. Una Agenda Digital para Europa. Comisión Europea. Bruselas, 26 de agosto de 2010.

Directiva 2009/140/CE, por la que se modifican la Directiva 2002/21/CE relativa a un marco regulador común de las redes y los servicios de comunicaciones electrónicas, la Directiva 2002/19/CE relativa al acceso a las redes de comunicaciones electrónicas y recursos asociados, y a su interconexión, y la Directiva 2002/20/CE relativa a la autorización de redes y servicios de comunicaciones electrónicas. 
Green Paper on Services of General Interest. Commission of the European Communities. Bruselas, 21.5.2003. COM(2003) 270 final. Disponible en: http://europa.eu/ legislation_summaries/competition/state_aid/123013_en.htm

Ley $3{ }^{\text {a }}$ de 4 de junio de 2013, de creación de la Comisión Nacional de los Mercados y la Competencia.

Ley 9. ${ }^{\text {a de }} 9$ de mayo de 2014, General de Telecomunicaciones.

Ortiz LAVERDE, SANDRA MiLena. (2014). Entre regulación y competencia: revisión institucional de la Comisión Nacional de los Mercados y la Competencia - CNMC. (Memoria para optar para el título de Doctor). Universidad Complutense de Madrid.

Presentación de Proyecto de la nueva ley de telecomunicaciones hecha por el Ministerio de Industria, Energía y Turismos. Madrid. Septiembre 2013. Disponible en: http://www.minetur.gob.es/es-es/gabineteprensa/notasprensa/2013/documents/ leygeneraltelecomunicaciones.pdf 


\section{ANEXOS}

5.1. Cuadro de traslado de competencias de la Comisión del Mercado de las Telecomunicaciones (boy Comisión Nacional de los Mercados y la Competencia) al Ministerio de Industria, Energía y Turismo.

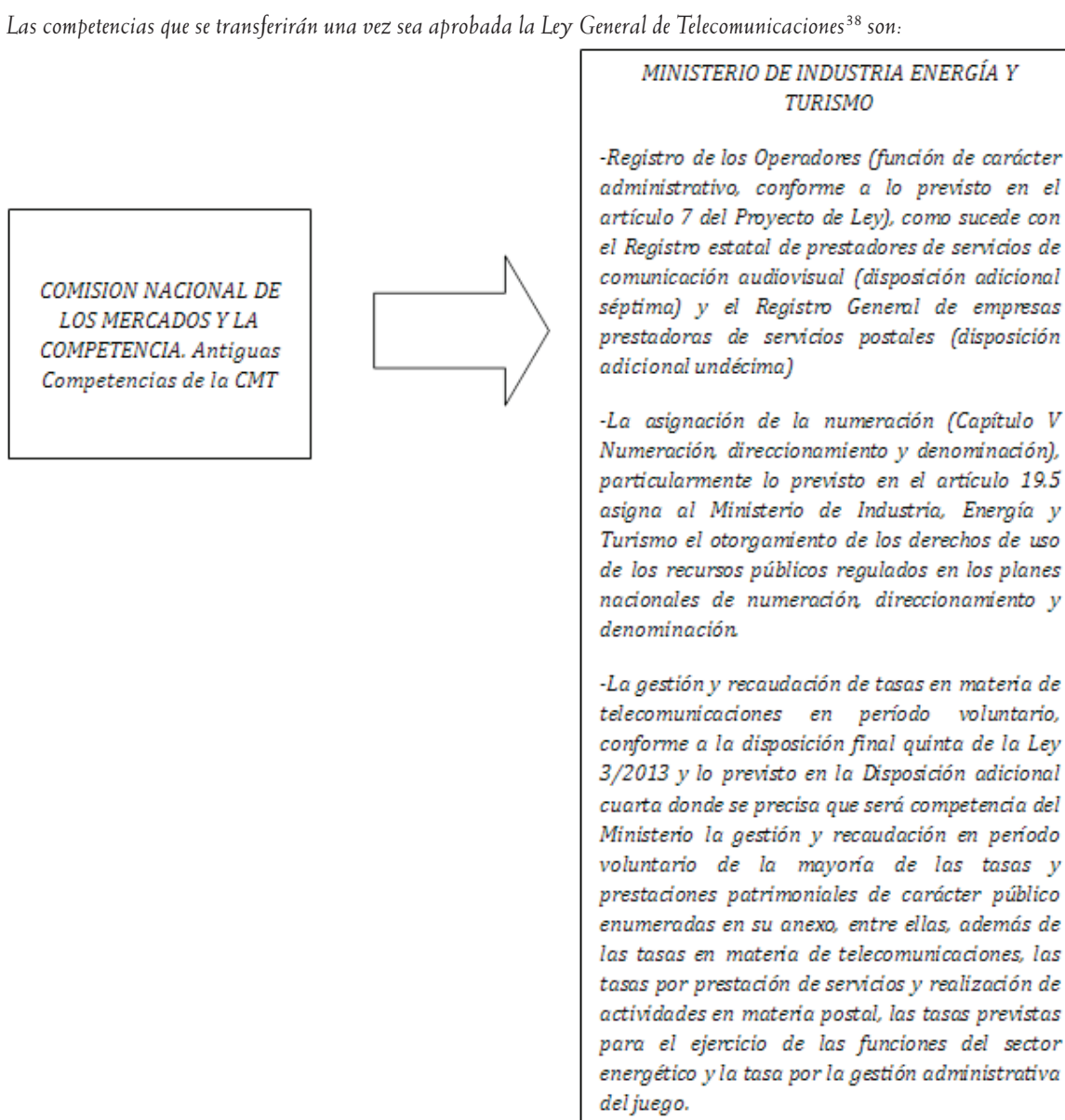

COMISION NACIONAL DE LOS MERCADOS Y LA COMPETENCIA. Antiguas Competencias de la CMT

-Registro de los Operadores (función de carácter administrativo, conforme a lo previsto en el artículo 7 del Proyecto de Ley), como sucede con el Registro estatal de prestadores de servicios de comunicación audiovisual (disposición adicional séptima) y el Registro General de empresas prestadoras de servicios postales (disposición adicional undécima)

- La asignación de la numeración (Capitulo V Numeración direccionamiento y denominación), particularmente lo previsto en el artículo 19.5 asigna al Ministerio de Industria, Energía y Turismo el otorgamiento de los derechos de uso de los recursos públicos regulados en los planes nacionales de numeración direccionamiento $y$ denominación

- La gestión y recaudación de tasas en materia de telecomunicaciones en período voluntario, conforme a la disposición final quinta de la Ley 3/2013 y lo previsto en la Disposición adicional cuarta donde se precisa que será competencia del Ministerio la gestión y recaudación en periodo voluntario de la mayoría de las tasas y prestaciones patrimoniales de carácter público enumeradas en su anexo, entre ellas, además de las tasas en materia de telecomunicaciones, las tasas por prestación de servicios y realización de actividades en materia postal, las tasas previstas para el ejercicio de las funciones del sector energético y la tasa por la gestión administrativa del juego.

Fuente: OrTIZ LAVERDE, SANDRA MILENA. (2014). Entre regulación y competencia: revisión institucional de la Comisión Nacional de los Mercados y la Competencia - CNMC ${ }^{39}$. Cuadro 72 - Disposiciones Adicionales de la Ley de Creación de la Comisión Nacional de los Mercados y la Competencia - Creación Propia: Transferencia de Funciones de la CMT al Ministerio de Industria, Energía y Turismo.

38 Vale la pena destacar lo precisado por parte de la Comisión del Mercado de Telecomunicaciones, respecto a la transferencia de funciones al Ministerio y que es recogido en el Dictamen del Consejo de Estado sobre el Anteproyecto de Ley General de Telecomunicaciones y los inconvenientes que generaría la asunción de competencias por un ente diferente al del organismo regulador. Ibíd., pp. 11 ss.

OrTiz Laverde (2014, pp. 619 ss.). 


\subsection{Cuadro de funciones del Ministerio de Industria, Energía y Turismo y la Comisión Nacio-} nal de Mercados y la Competencia (artículos 69 y 70, de la Ley 9. a de 9 mayo de 2014).

\begin{tabular}{|c|c|}
\hline Ministerio de Industria, Turismo y Energía & Comisión Nacional de Mercados y la Competencia \\
\hline $\begin{array}{l}\text { 1. Ejecutar la política en materia de servicios de } \\
\text { telecomunicaciones para la defensa nacional y la } \\
\text { protección civil que adopte el Gobierno. } \\
\text { 2. Ejercer las funciones en materia de la evaluación } \\
\text { de la conformidad de equipos y aparatos de tele- } \\
\text { comunicaciones. } \\
\text { 3. Ejercer las funciones atribuidas a la Administración } \\
\text { General del Estado en materia de autorización } \\
\text { e inspección de instalaciones radioeléctricas en } \\
\text { relación con los niveles únicos de emisión ra- } \\
\text { dioeléctrica. } \\
\text { 4. Ejercer sus competencias en los procedimientos de } \\
\text { licitación para la obtención de derechos de usos } \\
\text { del dominio público radioeléctrico. } \\
\text { 5. Elaborar estudios e informes y, en general, asesorar } \\
\text { a la Administración General del Estado en todo lo } \\
\text { relativo a la administración del dominio público } \\
\text { radioeléctrico. } \\
\text { 6. Elaborar proyectos de planes técnicos nacionales } \\
\text { de radiodifusión y televisión, y desarrollarlos. } \\
\text { 7. Formular proyectos de normas relativas a la infraes- } \\
\text { tructura común de comunicaciones electrónicas en } \\
\text { el interior de edificios y conjuntos inmobiliarios, } \\
\text { hacer seguimiento a su implantación en España. } \\
\text { 8. Garantizar el cumplimiento de compromisos in- } \\
\text { ternacionales en materia de telecomunicaciones. } \\
\text { 9. Garantizar el cumplimiento de la normativa sobre } \\
\text { datos personales y protección de la intimidad en } \\
\text { el sector de las comunicaciones electrónicas }{ }^{40} \text {. } \\
\text { 10. Gestionar el Registro de Operadores, el Registro } \\
\text { de empresas instaladoras de telecomunicación, y } \\
\text { el Registro Público de Radiofrecuencias }{ }^{41} \text {. } \\
\text { 11. Gestionar la asignación de los recursos órbita- } \\
\text { espectro para comunicaciones por satélite. } \\
\text { 12. Gestionar, en periodo voluntario, las tasas en } \\
\text { materia de telecomunicaciones a que se refiere la } \\
\text { Ley } 9 \text { a de } 09 \text { de mayo de } 2014 \text {. } \\
\text { 13. Gestionar, liquidar, inspeccionar y recaudar, en } \\
\text { periodo voluntario, los aportes para la financiación } \\
\text { de Radio y Televisión Española }\end{array}$ & $\begin{array}{l}\text { 1. Decidir la imposición, como medida excepcional, } \\
\text { de la obligación de separación funcional a los } \\
\text { operadores con poder significativo en el mercado } \\
\text { integrados verticalmente. } \\
\text { 2. Decidir la imposición de obligaciones a los ope- } \\
\text { radores que dispongan de interfaces de progra- } \\
\text { ma de aplicaciones (API) y guías electrónicas de } \\
\text { programación (EPG) para que faciliten el acceso } \\
\text { a estos recursos, en la medida que sea necesario } \\
\text { para garantizar el acceso de los usuarios finales a } \\
\text { determinados servicios digitales de radiodifusión } \\
\text { y televisión. } \\
\text { 3. Definir y analizar los mercados de referencia } \\
\text { relativos a redes y servicios de comunicaciones } \\
\text { electrónicas y el ámbito geográfico de los mismos. } \\
\text { 4. Determinar la cuantía que supone el coste neto } \\
\text { en la prestación del servicio universal }{ }^{42} \text {. } \\
\text { 5. Establecer, cuando proceda, las obligaciones es- } \\
\text { pecíficas que correspondan a los operadores con } \\
\text { poder significativo en mercados de referencia. } \\
\text { 6. Establecer el procedimiento para cuantificar los } \\
\text { beneficios no monetarios obtenidos por el opera- } \\
\text { dor u operadores encargados de la prestación del } \\
\text { servicio universal. } \\
\text { 7. Fijar las características y condiciones para la con- } \\
\text { servación de los números. } \\
\text { 8. Fomentar y garantizar la adecuación del acceso, } \\
\text { la interconexión y la interoperabilidad de los } \\
\text { servicios de comunicaciones. } \\
\text { 9. Identificar qué operadores poseen un poder sig- } \\
\text { nificativo en el mercado cuando del análisis de } \\
\text { los mercados de referencia se constate que no se } \\
\text { desarrollan en un entorno de competencia efectiva. } \\
\text { 10. Participar en el proceso de elaboración de normas } \\
\text { que afecten a su ámbito de competencias en ma- } \\
\text { teria de comunicaciones electrónicas, a través de } \\
\text { la formulación de informes. } \\
\text { operadores de comunicaciones electrónicas }{ }^{43} \text {. }\end{array}$ \\
\hline
\end{tabular}

40 La normativa relativa a datos personales y protección de la intimidad en el sector de las comunicaciones electrónicas es la siguiente: Directiva 2009/136/CE; Directiva 2002/58/ CE; Directiva 97/46/CE; Reglamento (CE) 2006/2004.

41 El Registro Público de Radiofrecuencias será accesible a través de internet y en él constarán los titulares de concesiones administrativas para el uso privativo del dominio público radioeléctrico.

42 Entiéndase incluida la competencia de definir y revisar la metodología para determinar dicho coste neto.

43 Ley 60 de 23 de diciembre 2003, sobre Arbitraje. 


\begin{tabular}{|c|c|}
\hline Ministerio de Industria, Turismo y Energía & Comisión Nacional de Mercados y la Competencia \\
\hline $\begin{array}{l}\text { hechos por los operadores de telecomunicaciones } \\
\text { y los prestadores privados del servicio de comu- } \\
\text { nicación audiovisual televisiva }{ }^{44} \text {. } \\
\text { 14. Hacerse partícipe en los organismos internaciona- } \\
\text { les relacionados con la planificación del espectro } \\
\text { radioeléctrico. } \\
\text { 15. Proponer al Gobierno la aprobación de planes } \\
\text { nacionales de numeración, direccionamiento y } \\
\text { denominación, y el otorgamiento de los derechos } \\
\text { de uso de los recursos públicos regulados en dichos } \\
\text { planes. } \\
\text { 16. Proponer al Gobierno políticas que faciliten el } \\
\text { desarrollo y evolución de las obligaciones de } \\
\text { servicio público. } \\
\text { 17. Proponer al Gobierno políticas para el reconoci- } \\
\text { miento y garantía de los derechos y obligaciones } \\
\text { de carácter público en la explotación de redes y } \\
\text { en la prestación de servicios de comunicaciones } \\
\text { electrónicas así como los derechos de los usuarios } \\
\text { finales. } \\
\text { 18. Proteger el dominio público radioeléctrico }{ }^{45} \text {. } \\
\text { 19. Realizar el control y seguimiento de las obliga- } \\
\text { ciones de servicio público que correspondan a los } \\
\text { distintos operadores en la explotación de redes } \\
\text { o la prestación de servicios de comunicaciones } \\
\text { electrónicas. } \\
\text { 20. Realizar la comprobación técnica de emisiones ra- } \\
\text { dioeléctricas para (i) identificar, localizar y eliminar } \\
\text { las interferencias, infracciones, irregularidades y } \\
\text { perturbaciones de los sistemas de radiocomuni- } \\
\text { cación, y (ii) verificar el uso efectivo y eficiente } \\
\text { del dominio público radioeléctrico por parte de } \\
\text { atribuidas por ley o por real decreto. } \\
\text { los titulares de derechos de uso. } \\
\text { 21. Realizar la propuesta de planificación, la gestión } \\
\text { y el control del dominio público radioeléctrico, } \\
\text { así como la tramitación y el otorgamiento de los } \\
\text { títulos habilitantes para su utilización. } \\
\text { 22. Realizar las funciones atribuidas de manera expresa } \\
\text { por la norma comunitaria, la presente ley y su } \\
\text { anarivana desarollo. }\end{array}$ & $\begin{array}{l}\text { 12. Resolver los conflictos en los mercados de comu- } \\
\text { nicaciones electrónicas }{ }^{46} \text {. } \\
\text { 13. Ser consultada por el Gobierno y el Ministerio } \\
\text { de Industria, Energía y Turismo en materia de } \\
\text { comunicaciones electrónicas, particularmente en } \\
\text { aquellas materias que puedan afectar el desarrollo } \\
\text { libre y competitivo del mercado. } \\
\text { 14. Ser consultada en materia de comunicaciones } \\
\text { electrónicas por las comunidades autónomas y } \\
\text { las corporaciones locales. } \\
\text { 15. Realizar las funciones atribuidas de manera expresa } \\
\text { por la normativa comunitaria, la presente ley y su } \\
\text { normativa de desarrollo. } \\
\text { 16. Realizar cualesquiera otras funciones que le sean } \\
\text { atribuidas por ley o por real decreto. }\end{array}$ \\
\hline
\end{tabular}

44 Ley 8 . $^{\text {a de }} 28$ de agosto de 2009. Artículos 5 y 6.

45 Para el desarrollo de esta función, el Ministerio de Industria, Energía y Turismo podrá realizar emisiones en aquellas frecuencias y canales radioeléctricos cuyos derechos de uso, en el ámbito territorial correspondiente, no hayan sido otorgados.

46 La ley hace especial mención a los conflictos entre operadores relacionados con: (a) la obligación de utilización compartida del dominio público o la propiedad privada ubicación compartida de infraestructuras y recursos asociados ${ }_{i}$ (c) el acceso a infraestructuras susceptibles de alojar redes públicas de comunicaciones electrónicas. y (d) el acceso a las redes de comunicaciones electrónicas titularidad de los órganos o entes gestores de infraestructuras de transporte de competencia estatal. 